\title{
Shell Effects on Rotational Damping in Superdeformed Nuclei
}

\author{
K. Yoshida and M. Matsuo ${ }^{a}$, \\ Research Center for Nuclear Physics, Osaka University, Ibaraki, Osaka, Japan \\ ${ }^{a}$ Yukawa Institute for Theoretical Physics, Kyoto University, Kyoto 606-01, Japan
}

\begin{abstract}
Damping of collective rotational motion in $A \sim 190$ and $A \sim 150$ superdeformed nuclei is studied by means of the cranked shell model with two-body residual force. Numerical calculations predict that in a typical $A \sim 190$ superdeformed nucleus, ${ }^{192} \mathrm{Hg}$, the rotational damping width is significantly small, $\Gamma_{\text {rot }} \sim 30 \mathrm{keV}$, and that the number of superdeformed bands in the off-yrast region amounts up to 150 at a given rotational frequency. These features are quite different from the prediction for $A \sim 150$ superdeformed nuclei and rare-earth normally deformed nuclei. It is shown that the single-particle alignments of the cranked Nilsson orbits have strong shell oscillation. It affects significantly the properties of rotational damping in superdeformed ${ }^{192} \mathrm{Hg}$.
\end{abstract}

PACS : 21.10.Ma, 21.10.Pc

keywords : Rotational damping, Superdeformed nuclei, Shell effect, Angular momentum alignment

\section{Introduction}

Recent progress in $\gamma$-ray spectroscopy has enabled us to study off-yrast levels and associated E2 transitions in rapidly rotating nuclei. The observed rotational bands lie near the yrast line and they are well described as quasi-particle or particle-hole excitations in a rotating mean field. Excited states which lie at higher intrinsic excitation energy above the yrast line are hardly observed by means of discrete $\gamma$-ray spectroscopy not only because individual levels are populated very weakly but also because they do not necessarily form the rotational band structure due to damping of collective rotational motion [1, 2]. The damping of collective rotational motion takes place in the energy region where density of many-particle many-hole states in the rotating mean field is high and the residual interaction causes configuration mixing among them. If the rotational damping sets in, E2 decay strength from an excited state spreads out over many final states. The number of rotational bands existing in a given nucleus is thus limited. The rotational damping has recently been studied experimentally through the statistical analyses of the quasi-continuum $\gamma$-ray spectra emitted from high spin off-yrast states. The fluctuation analysis method(FAM) [3] which extracts effective number of E2 decay paths revealed that in $A \sim 170$ rare-earth normally deformed nuclei there are only about 30 rotational band structures at a given rotational frequency and the rotational damping sets in at around $0.8 \mathrm{MeV}$ above yrast line. The occurence of rotational damping is reproduced by a microscopic realistic calculation, which takes into account the configuration mixing in the rotating mean field by means of the shell model diagonalization method [4, 5.

It has been argued theoretically that a strong shell effect is expected on the rotational damping in superdeformed nuclei [4] [6] since the presence of the shell gap in the single particle spectrum decreases dramatically the level density of excited many-particle many-hole configurations. This shell effect influences the intrinsic excitation energy where the rotational damping sets in. In a previous paper [6] we performed a systematic analysis for superdeformed nuclei in $A \sim 150$ region by means of the cranked shell model approach and predicted that the onset energy of damping and number of rotational bands are higher/larger than in $A \sim 170$ normally deformed nuclei, i.e., $E_{\text {onset }}=2 \sim 3$ 
$\mathrm{MeV}$ and $N_{\text {band }}=30 \sim 100$, and they vary significantly as functions of neutron and proton numbers due to the change in the shell gap. The continuum E2 $\gamma$-ray spectra associated with superdeformed states are observed experimentally in ${ }^{152} \mathrm{Dy}[7],{ }^{143} \mathrm{Eu}[8]$ and ${ }^{192} \mathrm{Hg}[9]$. So far the FAM analysis has been applied to ${ }^{143} \mathrm{Eu}[8]$ for which the theoretical prediction gives a qualitatively consistent account of the number of rotational bands $N_{\text {band }}$.

In this paper we will reveal that in addition to the level density effect there exists another kind of shell effect which affects significantly the rotational damping properties, especially the damping width, which is another fundamental quantity characterizing the rotational damping besides $N_{\text {band }}$ and $E_{\text {onset }}$. To demonstrate this, we investigate in detail off-yrast superdeformed states and associated rotational damping properties in a typical superdeformed nucleus ${ }^{192} \mathrm{Hg}$ in the $A \sim 190$ region, and make comparative study with $A \sim 150$ superdeformed nuclei and a typical normally deformed rare-earth nucleus ${ }^{168} \mathrm{Yb}$. The shell effect discussed here is originated from a shell structure associated with the angular momentum alignment of the single-particle orbits in the cranked mean field. As was discussed previously [10] highly aligned single-particle orbits affect the dispersion in rotational frequency of cranked many-particle many-hole states, which is a key quantity governing the rotational damping width. In this paper, we will show that because of the new shell effect the rotational damping width in superdeformed ${ }^{192} \mathrm{Hg}$ becomes remarkably small. As a consequence, the mechanism of onset of rotational damping in superdeformed ${ }^{192} \mathrm{Hg}$ becomes slightly different from that in $A \sim 150$ superdeformed and $A \sim 170$ normally deformed nuclei.

To this end, we perform a fully microscopic description of the superdeformed states in the high spin off-yrast region of ${ }^{192} \mathrm{Hg}$ by means of the shell model method based on the cranked mean field [5]. Since the formulation is essentially the same as the previous paper [6], it is described only briefly in Sec.2. In Sec.3 we show results obtained by our numerical calculation and characterize the onset of rotational damping and rotational damping width for ${ }^{192} \mathrm{Hg}$. In Sec. to understand mechanism of striking features in rotational damping in ${ }^{192} \mathrm{Hg}$ in terms of the shell effect associated with the single particle alignment structure. In Sec fa we will make a summary on the shell effects on rotational damping in superdeformed nuclei clarified in the previous and present investigations.

\section{Formulation}

To describe excited superdeformed states in the off-yrast region in ${ }^{192} \mathrm{Hg}$, we first construct unperturbed basis wave functions which are defined as multi-particle multi-hole configurations generated upon diabatic single particle routhian orbits [11] of cranked Nilsson mean-field Hamiltonian $h^{\omega}=h_{\mathrm{Nilsson}}-\omega j_{x}$. Here deformation parameters of the Nilsson potential are determined by means of Strutinsky minimization scheme [12] with the pairing correlation energy included. We consider quadrupole and hexadecupole deformation parameters $\left(\epsilon_{2}, \epsilon_{4}\right)$. We determine equilibrium deformation at zero rotational frequency. The shell correction energy for the pairing correlation is included in a standard way 13], $E^{\text {pair }}=-(P-\tilde{P})$, where $P$ is pairing correlation energy gain obtained by the BCS approximation and $\tilde{P}$ is the smoothed part obtained with equidistant single particle levels. Single particle levels within $\pm 6 \mathrm{MeV}$ from the Fermi surface are considered. The pairing coupling constant $G$ is determined so that the pairing gap for the equidistant model gives $12 / A^{-1 / 2} \mathrm{MeV}$. The total energy, including the liquid drop energy, shell correction energy and $E^{\text {pair }}$, evaluated at zero rotational frequency is minimized with respect to $\epsilon_{2}$ and $\epsilon_{4}$. Thus we obtained the equilibrium deformation parameters $\left(\epsilon_{2}, \epsilon_{4}\right)=(0.451,0.0115)$ for superdeformed state in ${ }^{192} \mathrm{Hg}$. The same deformation parameters are used to construct the basis states at high spins because the deformation is considered to change only slightly with increasing spin.

Total energy of a basis $n \mathrm{p}-n \mathrm{~h}$ configuration $\mu$ is expressed as

$$
E_{\mu}(I)=\sum_{\substack{\text { occupied } n \\ \text { in } \mu}} e_{n}\left(\omega_{I}\right)+\omega_{I} I+\frac{1}{2 \mathcal{J}_{\mu}^{\text {micro }}}\left(I-J_{x \mu}\left(\omega_{I}\right)\right)^{2}-E_{\text {smooth }}(I)+E^{\mathrm{RLD}}(I),
$$




$$
J_{x \mu}=\sum_{\substack{\text { occupied } n \\ \text { in } \mu}} i_{n}\left(\omega_{I}\right)
$$

where $e_{n}\left(\omega_{I}\right)$ is single particle routhian energy for the cranked Nilsson Hamiltonian. $J_{x \mu}\left(\omega_{I}\right)$ and $\mathcal{J}_{\mu}^{\text {micro }}$ are expectation value of the angular momentum operator $\hat{J}_{x}$ and dynamic moment of inertia, respectively, for the many-body configuration $|\mu(I)\rangle$. $i_{n}\left(\omega_{I}\right)$ is the $\hat{j}_{x}$ expectation value for the cranked Nilsson single particle orbits. Here reference rotational frequency $\omega_{I}$ is determined so that $J_{x}\left(\omega_{I}\right)=I$ is satisfied for the yrast configuration. $E^{\text {smooth }}$ and $E^{\mathrm{RLD}}$ are the smooth part and the rotating liquid drop energy [14], respectively.

As the residual two-body interaction we adopt the delta interaction, $v(1,2)=-v_{\tau} \delta\left(\vec{x}_{1}-\vec{x}_{2}\right)$, $v_{\tau}=340 \mathrm{MeVfm}^{3}$ for like nucleons and $v_{\tau}=500 \mathrm{MeVfm}^{3}$ for the neutron-proton interaction [15]. A shell model Hamiltonian matrix for the $n \mathrm{p}-n \mathrm{~h}$ basis states $\{|\mu(I)\rangle\}$ is constructed by including the residual two-body interaction and diagonalized numerically for each spin and parity. The obtained energy eigenstates are linear combinations of unperturbed basis,

$$
|\alpha(I)\rangle=\sum_{\mu} X_{\mu}^{\alpha}(I)|\mu(I)\rangle .
$$

The E2 transition strength between an initial state $\alpha$ at $I$ and a final state $\beta$ at $I-2$ is given by

$$
M_{\alpha I \beta I-2}^{2}=\frac{15}{128 \pi} Q_{0}^{2}\left|\sum_{\mu} X_{\mu}^{\beta *}(I-2) X_{\mu}^{\alpha}(I)\right|^{2},
$$

assuming that collective E2 transition takes place between the basis states having the same microscopic configuration and the static quadrupole moment $Q_{0}$ is constant for all configurations. Then

$$
w_{\alpha I \rightarrow \beta I-2}=\left|\sum_{\mu} X_{\mu}^{\beta *}(I-2) X_{\mu}^{\alpha}(I)\right|^{2}
$$

represents normalized transition strength satisfying $\sum_{\beta} w_{\alpha I \rightarrow \beta I-2}=1$.

The single particle levels located within $4.0 \mathrm{MeV}$ above/below the Fermi surface are taken into account as active orbits in constructing the many-body basis states, and up to five-particle five -hole configurations are included. The energetically lowest 2000 basis states (for each $I^{\pi}$ ) are chosen to diagonalize the Hamiltonian matrix. The model space of the diagonalization is not large enough to describe static pairing correlation which may be important for near-yrast states at low spins. It is to be noted, however, that the pairing correlation is weakened by the Coriolis anti-pairing effect at very high spin and also by the blocking effect for the excited superdeformed states in the off-yrast region.

In the following section we also show results for superdeformed states in ${ }^{143} \mathrm{Eu}$ and ${ }^{152} \mathrm{Dy}$, selected as representatives in the $A \sim 150$ region, and also normally deformed high spin states in a typical rare-earth nucleus ${ }^{168} \mathrm{Yb}$. The calculational procedure for those nuclei were already described in the previous publications 6 , 5 . The numerical results shown in this paper are obtained by using the same delta interaction as in ${ }^{192} \mathrm{Hg}$.

It is known that the superdeformed states usually coexist with weakly deformed states in the same nucleus at the same spin and parity. They do not mix, however, very strongly at high spin regions where there is a potential barrier between the two kinds of states and the level density of weakly deformed states is relatively small. To make comparison with experimental data including low spin region where the mixing may be effective, it is necessary to take into account the barrier penetration. The excited superdeformed states may decay not only via collective E2 but also via statistical E1 transition which becomes possible through the mixing of weakly deformed states [16, 17]. Since our aim in this paper is to discuss mechanism of rotational damping we leave this effect for future investigation.

\section{Results}




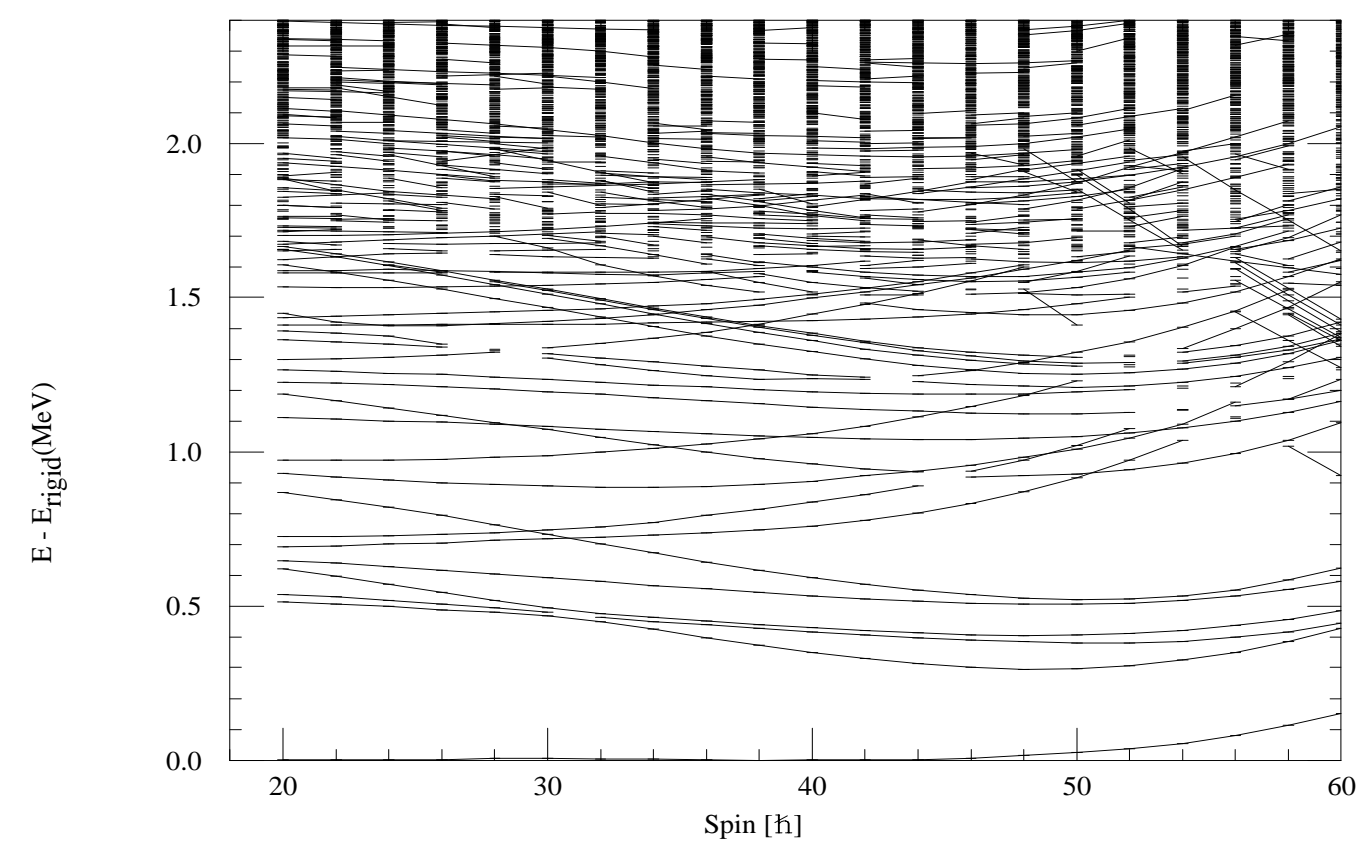

Figure 1: The calculated energy levels plotted with little horizontal bars for $(\alpha, \pi)=(0,+)$ states in superdeformed ${ }^{192} \mathrm{Hg}$. A rigid-rotor rotational energy $E_{\text {rigid }}=I(I+1) / 234(\mathrm{MeV})$ is subtracted. Solid lines connecting the energy levels represent strong E2 transitions with normalized strength larger than $\sqrt{1 / 2}=0.707$.

\subsection{Onset of rotational damping}

In this subsection, we discuss onset property of rotational damping by analyzing onset energy of rotational damping and number of superdeformed rotational bands. In Fig. 1 we display obtained superdeformed levels for parity $\pi=+$, signature $\alpha=0$ in ${ }^{192} \mathrm{Hg}$. Energy levels are shown by short horizontal bars. Strong E2 transitions satisfying $w_{\alpha I \rightarrow \beta I-2} \geq 0.707=\frac{1}{\sqrt{2}}$ are plotted with solid lines, which are discrete transitions forming the rotational band structure. The rigid-body rotor energy $E_{\text {rigid }}=I(I+1) / 234 \mathrm{MeV}$ is subtracted. The vertical axis indicates approximately the intrinsic excitation energy measured from the yrast line.

It is seen from Fig. 1. that there are apparently more than 20 rotational bands with even spin and parity in ${ }^{192} \mathrm{Hg}$, which lie between the yrast line and up to $E_{x} \sim 1.5 \mathrm{MeV}$ above yrast. As the excitation energy increases, the rotational band structure gradually disappears and the rotational damping sets in. To describe this transition more quantitatively, we calculate an effective number of E2 branches decaying from a state $\alpha$, which is defined by

$$
n_{\text {branch }}(\alpha)=\left(\sum_{\beta} w_{\alpha I \rightarrow \beta I-2}^{2}\right)^{-1} .
$$

Calculating the average value of $n_{\text {branch }}$ as a function of intrinsic excitation energy measured from the yrast line(by taking a energy bin of $0.1 \mathrm{MeV}$ interval), we define onset energy of damping $E_{\text {onset }}$ by the condition that $\left\langle n_{\text {branch }}\right\rangle\left(E_{\text {onset }}\right)=2$. In Fig.2(a), we show the obtained onset energy for ${ }^{192} \mathrm{Hg}$ as a function of angular momentum together with the results for $A \sim 150$ superdeformed nuclei and normally deformed ${ }^{168} \mathrm{Yb}$. The onset energy varies in superdeformed ${ }^{192} \mathrm{Hg}$ from 1.8 to $1.3 \mathrm{MeV}$ with increasing spin. It is apparently smaller than those for $A \sim 150$ superdeformed nuclei and larger than ${ }^{168} \mathrm{Yb}$.

We define number of rotational bands denoted by $N_{\text {band }}$ by counting at a given spin the levels 

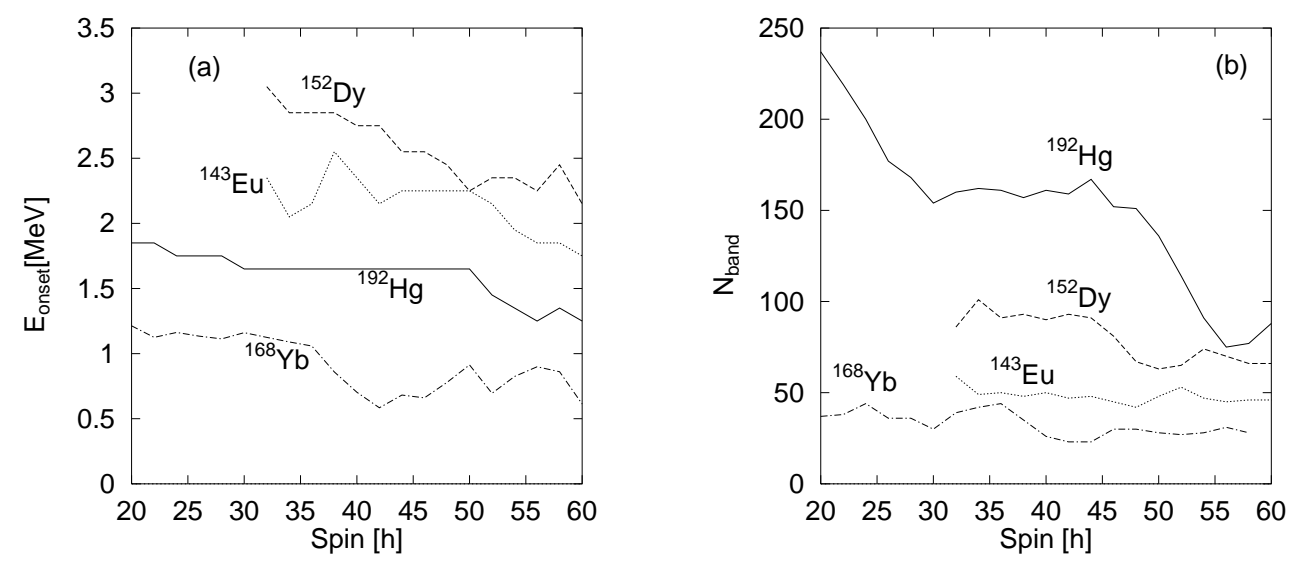

Figure 2: (a)The onset energy of rotational damping $E_{\text {onset }}$ for superdeformed ${ }^{192} \mathrm{Hg}$ as a function of spin and those for superdeformed ${ }^{152} \mathrm{Dy},{ }^{143} \mathrm{Eu}$ and normally deformed ${ }^{168} \mathrm{Yb}$. (b)The number of discrete rotational bands $N_{\text {band }}$ for the same nuclei.

for which the condition $n_{\text {branch }}(\alpha)<2$ is satisfied for two consecutive decay-in and decay-out E2 transitions. The numbers for four sets of signature and parity quantum numbers are summed up. This quantity corresponds to the experimental effective number of paths [3] which is extracted from the spectral fluctuation at the first ridge of the $E_{\gamma_{1}} \times E_{\gamma_{2}}$ spectra, which are formed by two consecutive E2 transitions along rotational bands. The calculated number of rotational bands is shown in Fig.2(b) as a function of angular momentum. A noticeable feature is that the number of superdeformed rotational bands in ${ }^{192} \mathrm{Hg}$ amounts up to about 150 on average, which is much larger than in the $A \sim 150$ superdeformed nuclei (see also Fig.9 in Ref.[6] ) and typical value 30 of $N_{\text {band }}$ in normally deformed nuclei. Dependence of the number of bands on spin is also distinct in ${ }^{192} \mathrm{Hg}$; it is about 250 at $I=20 \hbar$ and decreases as spin increases and takes 150 at $30 \hbar \leq I \leq 46 \hbar$ and again begins to decrease at $I=48 \hbar$ and reaches less than 100 at $I \geq 54 \hbar$.

The quasi-continuum E2 $\gamma$ rays associated with superdeformed states are observed in ${ }^{192} \mathrm{Hg}$ in the spin region $I \sim 40 \hbar$ [9], but there is no direct experimental information, such as $N_{\text {band }}$, concerning the onset of rotational damping.

\subsection{Rotational damping width}

In this subsection we discuss in detail the damping width of collective rotational motion. As excitation energy exceeds the onset energy of damping, E2 strength decaying from an excited state is fragmented over several or more final states and distributed within a certain interval of $\gamma$-ray energy $E_{\gamma}$, corresponding to the rotational damping width $\Gamma_{\text {rot }}$. The distribution is expressed by a single-step E2 strength function defined by

$$
S^{(1)}\left(E_{\gamma}\right)=\sum_{\alpha, \beta}^{\prime} w_{\alpha I \rightarrow \beta I-2} \delta\left(E_{\gamma}-E_{\alpha I}+E_{\beta I-2}\right) / \sum_{\alpha}^{\prime}
$$

where the summation of $\alpha$ runs over the initial states at $I$ in an energy bin, whereas $\beta$ runs over all final states at $I-2$. Figure 3 shows the calculated E2 strength distribution $S^{(1)}\left(E_{\gamma}\right)$ associated with an ensemble in an energy bin composed of 201st to 300th levels at each $I^{\pi}$ and averaged over parities. This ensemble of levels corresponds to the energy region, $E_{x} \approx 3.8,3.6$ and $2.4 \mathrm{MeV}$ above yrast for ${ }^{143} \mathrm{Eu},{ }^{152} \mathrm{Dy}$ and ${ }^{192} \mathrm{Hg}$, respectively, which is much higher than the onset energy by about 1-2 MeV. The distribution for lower energy bins(e.g., those of 1st-100th and 101st-200th) is not very different from that shown in Fig.).

The calculated strength distribution has the centroid corresponding to $2 \hbar \omega_{\text {rot }}=\frac{2 I}{\mathcal{J}}$ ( $\mathcal{J}$ being the moment of inertia) and the distribution width is broadened with increasing spin. The distributions 

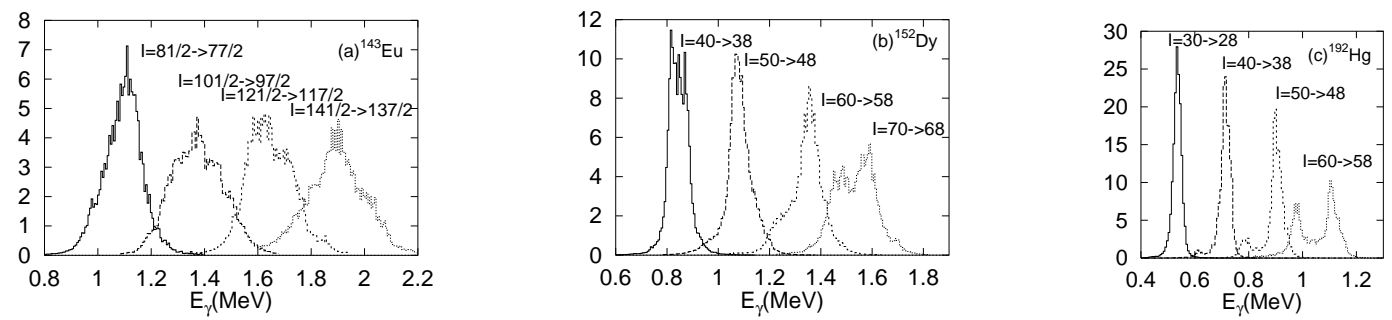

Figure 3: The single-step E2 strength distribution $S^{(1)}\left(E_{\gamma}\right)$ for transition $I \rightarrow I-2$ calculated for an energy bin composed of lowest 201-300th levels for each $I^{\pi}$ spectrum for superdeformed ${ }^{143} \mathrm{Eu}$, ${ }^{152} \mathrm{Dy}$ and ${ }^{192} \mathrm{Hg}$.

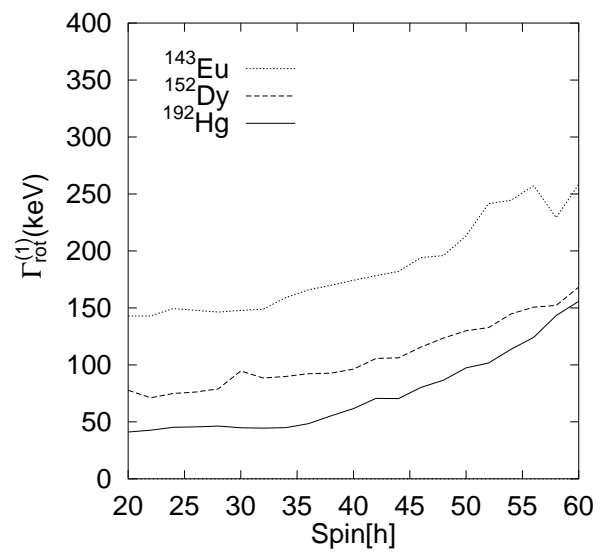

Figure 4: The rotational damping width $\Gamma_{\text {rot }}^{(1)}$ extracted from standard deviation of the single-step E2 strength distribution $S^{(1)}\left(E_{\gamma}\right)$ associated with the 201-300th levels, averaged over both parities and signatures, for ${ }^{192} \mathrm{Hg}$ and $A \sim 150$ superdeformed nuclei as a function of spin.

in ${ }^{152} \mathrm{Dy}$ and ${ }^{192} \mathrm{Hg}$ are split into two peaks at very high spins. This splitting comes from high- $j$ intruder orbits coming down close to the Fermi surface with increasing spin. Since the configurations occupying the high- $j$ orbit $\pi 7_{1}$ for ${ }^{152} \mathrm{Dy}$ or $\pi 8_{1}$ for ${ }^{192} \mathrm{Hg}$, have larger angular momentum alignment by sizable amount than the other ones, transitions associated with configurations occupying or excluding the high- $j$ orbits form peaks at different $\gamma$-ray energies.

It is useful to extract rotational damping width from the E2 distributions. However, it is difficult to define the FWHM because of the two-peak structure of the distribution at higher spins. An alternative definition may be given by the standard deviation $\sigma$ of the strength distribution $S^{(1)}\left(E_{\gamma}\right)$ and we define the width $\Gamma_{\text {rot }}^{(1)}$ by $2 \sigma$. The evaluated $\Gamma_{\text {rot }}^{(1)}$ averaged over both signatures and parities is shown in Fig. 1 . It is noted that $\Gamma_{\text {rot }}^{(1)}$ in ${ }^{192} \mathrm{Hg}$ is quite small $\Gamma_{\text {rot }}^{(1)} \lesssim 50 \mathrm{keV}$ at lower spins and increases with increasing spin. On the other hand $\Gamma_{\text {rot }}^{(1)}$ for ${ }^{143} \mathrm{Eu}$ is $150 \sim 250 \mathrm{keV}$, which is as large as for $A \sim 170$ normally deformed nuclei [5]. That for ${ }^{152}$ Dy is $90 \sim 150 \mathrm{keV}$, which is sizably smaller than ${ }^{143} \mathrm{Eu}$ though it is twice as large as that for ${ }^{192} \mathrm{Hg}$. We remark that the quantity $\Gamma_{\text {rot }}^{(1)}$ may overestimate the rotational damping width when the rotational damping is not well developed. For example, if it is calculated for energy bins of near-yrast levels below the onset energy of damping, $\Gamma_{\text {rot }}^{(1)}$ represents statistical dispersion of undamped E2 $\gamma$-ray energies which differ from band to band while the rotational damping width should be zero.

Another way to extract the rotational damping width may be derived from correlation between two consecutive rotational E2 transitions since the correlation distinguishes the damped transitions 

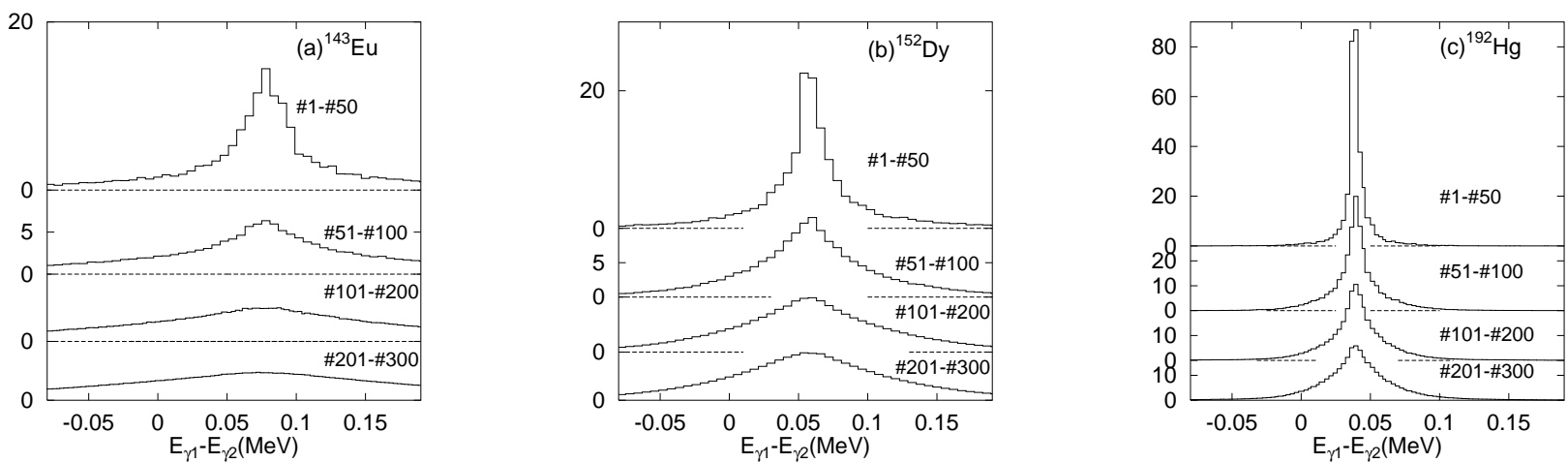

Figure 5: The two-step E2 strength distribution $S^{(2)}\left(E_{\gamma 1}-E_{\gamma 2}\right)$ associated with consecutive transitions $I \rightarrow I-2 \rightarrow I-4$ for different ensembles composed of different levels. It is averaged over spin interval $I=34-47 \hbar$ for ${ }^{192} \mathrm{Hg}$, and $I=44(89 / 2)-57(115 / 2) \hbar$ for ${ }^{152} \mathrm{Dy}\left({ }^{143} \mathrm{Eu}\right)$.

and rotational band structure more clearly. This idea is adopted also in the analysis of the experimental $E_{\gamma} \times E_{\gamma}$ spectra [18, 19]. The correlation can be represented by a function,

$$
S^{(2)}\left(E_{\gamma 1}-E_{\gamma 2}\right)=\sum_{\alpha \alpha^{\prime} \alpha^{\prime \prime}}^{\prime} w_{\alpha I \rightarrow \alpha^{\prime} I-2} w_{\alpha^{\prime} I-2 \rightarrow \alpha^{\prime \prime} I-4} \delta\left(E_{\gamma 1}-E_{\gamma 2}-E_{\alpha I}+2 E_{\alpha^{\prime} I-2}-E_{\alpha^{\prime \prime} I-4}\right) / \sum_{\alpha}^{\prime}
$$

which we call the two-step strength function. Here the summation runs over two consecutive transitions, i.e., the decay-in transitions from $\alpha$ at $I$ to $\alpha^{\prime}$ at $I-2$ and decay-out transitions from $\alpha^{\prime}$ to $\alpha^{\prime \prime}$ at $I-4$. An average is taken over levels $\alpha^{\prime}$ in an energy bin. If we assume a simple Lorentzian distribution for the first transition $I \rightarrow I-2$ and the second one $I-2 \rightarrow I-4, S^{(2)}$ become a convolution of the two Lorentzian distributions and the FWHM of $S^{(2)}$ is equal to twice of the rotational damping width. Thus it will be possible to define the rotational damping width as a half value of FWHM of $S^{(2)}$. We use different notation $\Gamma_{\text {rot }}^{(2)}$ for this quantity to distinguish it from $\Gamma_{\text {rot }}^{(1)}$.

The calculated two-step strength function $S^{(2)}$ shown in Fig. 5 does not necessarily show a simple Lorentzian nor Gaussian shape for the energy bin covering the onset region of damping. This is because there coexist undamped transitions which form rotational band structure, scars of rotational bands embedded in the damped region (they contribute to the narrow distribution) and fragmented damped transitions (producing wide distribution with width of $2 \times \Gamma_{\text {rot }}^{(2)}$ [20, 21, 20. As the excitation energy increases the wide component dominates as seen for the energy bin covering the 201st-300th levels. From this energy bin we extract the rotational damping width $\Gamma_{\text {rot }}^{(2)}$, which is plotted in Fig. 6 . It is noted that the two-step strength function $S^{(2)}$ has smooth profile in contrast to the two-peak behavior in the single-step strength function $S^{(1)}$. For this reason and the possible overestimate in $\Gamma_{\text {rot }}^{(1)}$, we refer mainly to $\Gamma_{\text {rot }}^{(2)}$ as the rotational damping width $\Gamma_{\text {rot }}$ in the following discussion.

It is seen from Fig. 6 that the rotational damping width $\Gamma_{\text {rot }}^{(2)}$ is $25 \sim 30 \mathrm{keV}$ for ${ }^{192} \mathrm{Hg}, 30 \sim 70 \mathrm{keV}$ for ${ }^{152} \mathrm{Dy}$ and $70 \sim 140 \mathrm{KeV}$ for ${ }^{143} \mathrm{Eu}$. Noticeable features are that the rotational damping width in ${ }^{192} \mathrm{Hg}$ is smaller by more than factor 4 than that in ${ }^{143} \mathrm{Eu}$, and that there is factor 2 difference between ${ }^{143} \mathrm{Eu}$ and ${ }^{152} \mathrm{Dy}$ in spite of similar mass number and deformation. These features are also seen in $\Gamma_{\text {rot }}^{(1)}$. So far there seems no experimental analysis that extracts directly rotational damping width associated with superdeformed states.

\section{Discussion}

In this section we discuss in detail the numerical results shown in the previous section with focuses on two distinctive features found in superdeformed ${ }^{192} \mathrm{Hg}$, i.e., (i) very small rotational damping width, $\Gamma_{\text {rot }} \sim 20-30 \mathrm{keV}$ and (ii) quite large number of rotational bands, $N_{\text {band }} \sim 150$. 


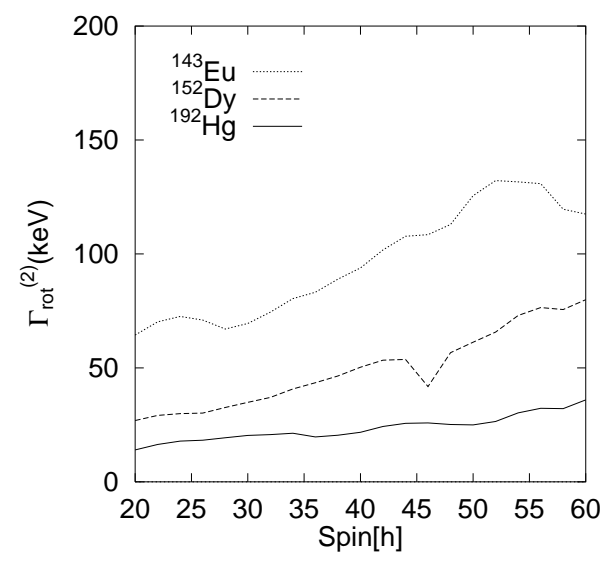

Figure 6: The rotational damping width $\Gamma_{\text {rot }}^{(2)}$ extracted from FWHM of the two-step E2 strength distribution $S^{(2)}\left(E_{\gamma 1}-E_{\gamma 2}\right)$ associated with 201-300th levels as a function of spin.

\subsection{Small rotational damping width and shell effect in alignments}

In order to understand the small rotational damping width in ${ }^{192} \mathrm{Hg}$, it is instructive to recall the discussion by Lauritzen et.al 2 on the basic features of rotational damping width. The unperturbed basis rotational bands, i.e., $n \mathrm{p}-n \mathrm{~h}$ configurations in the cranked mean-field potential, have different rotational frequencies at a given spin value, depending on intrinsic particle and hole configurations. Once the residual interaction is taken into account, it causes mixing among the unperturbed rotational bands and an energy eigenstate becomes a superposition of different basis states at the same spin. Under these circumstances, the rotational damping width is estimated to be

$$
\Gamma_{\text {rot }} \sim 4 \Delta \omega
$$

in terms of the statistical dispersion of E2 transition energies $\delta E_{\mu}=E_{\mu}(I)-E_{\mu}(I-2) \approx 2 \omega_{\mu}$ associated with basis bands $\mu$ or dispersion $\Delta \omega$ of rotational frequencies $\omega_{\mu}$ if the spreading width of basis states (due to the configuration mixing) $\Gamma_{\mu}$ is relatively small compared with the dispersion of rotational frequencies $\Delta \omega\left(\Gamma_{\mu}<2 \Delta \omega\right)$. In the other case $\left(\Gamma_{\mu}>2 \Delta \omega\right)$, the rotational damping width is estimated as

$$
\Gamma_{\text {rot }} \sim 2 \frac{(2 \Delta \omega)^{2}}{\Gamma_{\mu}}
$$

which is smaller than the estimate Eq.(9) (i.e., the motional narrowing). In any case, the damping width depends strongly (linearly or quadruticly) on the dispersion of rotational frequency $\Delta \omega$.

The dispersion of rotational frequency may be evaluated in two ways. A direct way is just to calculate numerically the variance of $\mathrm{E} 2$ transition energies $\delta E_{\mu}$ associated with the basis states $\mu$ obtained in the microscopic calculation. Namely,

$$
\Delta \omega_{\text {direct }}=\frac{1}{2} \sqrt{\left\langle\delta E_{\mu}^{2}\right\rangle-\left\langle\delta E_{\mu}\right\rangle^{2}}
$$

where the average is taken over an ensemble of states in an energy bin. In Fig. (7) (a) we show the calculated dispersion(times factor four) $4 \Delta \omega_{\text {direct }}$ for the lowest 200 levels at each spin and parity as a function of spin for the three superdeformed nuclei and a normally deformed nucleus ${ }^{168} \mathrm{Yb}$. We plot $4 \Delta \omega_{\text {direct }}$ since it corresponds to the simple analytic estimate of the rotational damping width (see Eq.(9)). The bin of lowest 200 levels has average excitation energy of $E_{x}=1.7,2.7,2.9$, and 1.8 $\mathrm{MeV}$ above the yrast line for ${ }^{168} \mathrm{Yb},{ }^{143} \mathrm{Eu},{ }^{152} \mathrm{Dy}$, and ${ }^{192} \mathrm{Hg}$, respectively. It covers the onset region of rotational damping. 

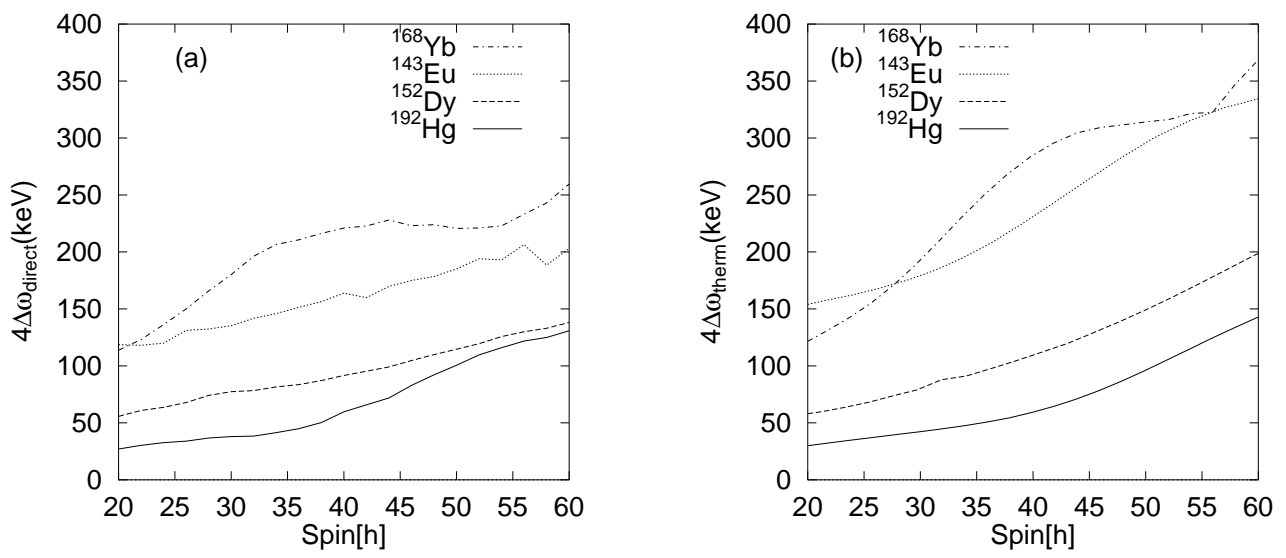

Figure 7: (a)The statistical dispersion of rotational frequency $\Delta \omega_{\text {direct }}$ which is calculated from the transition energy $\delta E_{\mu}=E_{\mu}(I)-E_{\mu}(I-2)$ for the $n$ p- $n$ h states covering the lowest 200 configurations for each $I^{\pi}$. Here $4 \Delta \omega_{\text {direct }}$ is plotted as a function of spin. (b)The dispersion of rotational frequency $\Delta \omega_{\text {therm }}$ obtained by means of the thermal grand canonical ensemble (Eq.(12)). The temperature of the ensemble is chosen so that its average excitation energy agrees with that of energy bin used to calculate $\Delta \omega_{\text {direct }}$. Namely, $T=0.31,0.46,0.48$ and $0.32 \mathrm{MeV}$ for ${ }^{168} \mathrm{Yb},{ }^{143} \mathrm{Eu},{ }^{152} \mathrm{Dy}$ and ${ }^{192} \mathrm{Hg}$, respectively.

Another approximate evaluation can be made by introducing a thermal grand canonical ensemble of excited configurations in terms of cranked Nilsson orbits 10. This gives

$$
\Delta \omega_{\text {therm }}=\frac{1}{\mathcal{J}} \sqrt{\sum_{n} i_{n}^{2} f_{n}\left(1-f_{n}\right)},
$$

where it is assumed that nuclear shape is stable and the moment of inertia $\mathcal{J}$ does not depend very much on intrinsic configurations (this can be justified in stably deformed nuclei). In this case dispersion in the rotational frequency $\omega_{\mu}$ of the $n \mathrm{p}-n \mathrm{~h}$ states is determined by the thermal fluctuation in the alignment $\sqrt{\sum_{n} i_{n}^{2} f_{n}\left(1-f_{n}\right)}$ which is expressed in terms of the single-particle alignments $i_{n}$ of the cranked Nilsson routhian orbits and the Fermi-Dirac distribution function $f_{n}=\left(1+\exp \frac{e_{n}-\lambda}{T}\right)^{-1}$ with $e_{n}$ and $\lambda$ being the single particle routhian energy and the Fermi energy. Calculated dispersion $4 \Delta \omega_{\text {therm }}$ is shown in Fig. (7). b). Here the temperature $T$ is chosen so that average excitation energy $\left\langle E_{x}\right\rangle_{\text {therm }}$ agrees with the average energy of the ensemble shown in Fig. 7 (a).

Characteristic features of $\Delta \omega_{\text {direct }}$ seen in Fig. [](a) are: 1) $\Delta \omega_{\text {direct }}$ varies quite significantly for different nuclei. It becomes the smallest $(<50 \mathrm{keV})$ in ${ }^{192} \mathrm{Hg}$ at not very large spins $(I \lesssim 40 \hbar)$, which is about four times smaller than that in ${ }^{143} \mathrm{Eu}$ and normally deformed ${ }^{168} \mathrm{Yb}$. It differs by about factor two between superdeformed ${ }^{143} \mathrm{Eu}$ and ${ }^{152} \mathrm{Dy}$ although the mass number and deformation is rather similar. 2) $\Delta \omega_{\text {direct }}$ increases as a function of spin (as can be expected from an analytic estimate [2], see also Eq.(17)), but it exhibits strong non-linear dependence on spin. It is noticed that $4 \Delta \omega_{\text {direct }}$ shown in Fig.] (a) agrees quite well with the "rotational damping width" $\Gamma_{\text {rot }}^{(1)}$, shown in Fig.4, which is estimated from the dispersion of the single-step E2 strength function $S^{(1)}\left(E_{\gamma}\right)$. The rotational damping width $\Gamma_{\text {rot }}^{(2)}$ extracted from the two-step correlation (Fig.6) also reflects the above characteristic features. The approximate estimate $\Delta \omega_{\text {therm }}$ based on the grand canonical ensemble, shown in Fig. 7 (b), gives essentially the same results as $\Delta \omega_{\text {direct }}$, and the two characteristic features discussed above are also seen. These observations indicate that the characteristic behaviors of the dispersion $\Delta \omega$ of rotational frequency and the rotational damping width $\Gamma_{\text {rot }}$ can be ascribed to properties of the single-particle orbits of the cranked Nilsson mean field, i.e., the routhian spectrum $e_{n}$ and single-particle alignments $i_{n}$.

To clarify the relation of $\Delta \omega$ to the cranked Nilsson single particle orbits, it is useful to introduce 

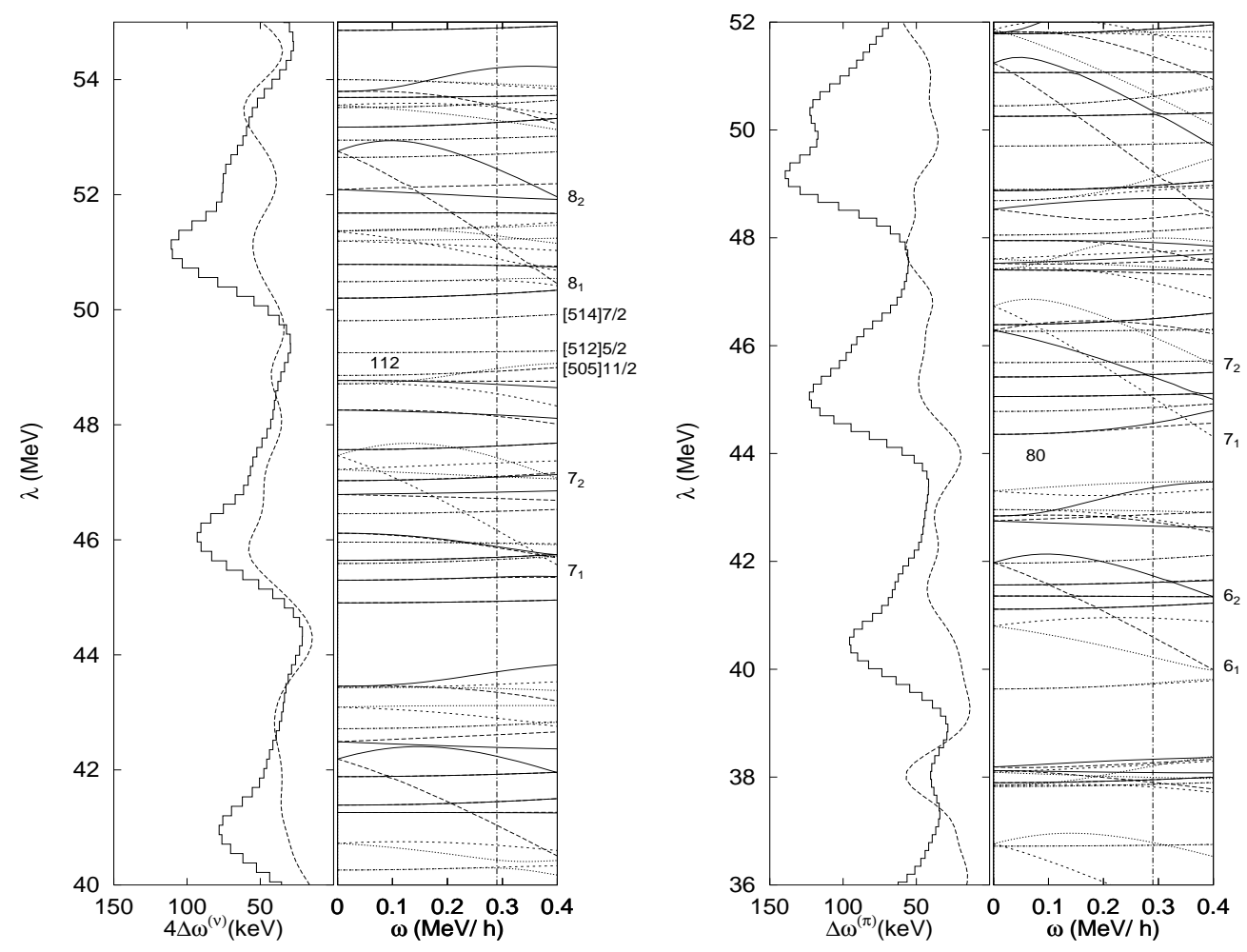

Figure 8: The neutron and proton contributions $\Delta \omega^{(\nu, \pi)}$ to the dispersion of rotational frequency as a function of the Fermi energy $\lambda$ for superdeformed ${ }^{192} \mathrm{Hg}$ (solid line). Temperature is chosen as $T=0.32 \mathrm{MeV}$ and rotational frequency $\omega=0.29 \mathrm{MeV} / \hbar$ corresponding to $I=40 \hbar$. The smoothed spectral density(dashed line) is also drawn in an arbitrary unit. The right panel shows the corresponding cranked Nilsson routhian diagram. The vertical line indicates $\omega=0.29 \mathrm{MeV} / \hbar$.

a quantity which represents density of the single-particle alignment as a function of routhian energy

$$
\rho_{\mathrm{al}}^{(\nu, \pi)}(e)=\sum_{n \in \nu, \pi} i_{n}^{2} \delta\left(e-e_{n}\right)
$$

which is defined separately for neutrons $(\nu)$ and $\operatorname{protons}(\pi)$. Here it is noted that the function $\frac{1}{T} f_{n}\left(1-f_{n}\right)=\frac{d}{d \lambda} f_{n}$ in the r.h.s of Eq.(12) is a smooth function peaked at the Fermi energy $\lambda$ with width of order of temperature $T$ (and normalized as $\int d \lambda \frac{d}{d \lambda} f_{n}=1$ ). This allows us to regard the function $\frac{d}{d \lambda} f_{n} \equiv g\left(\lambda-e_{n}\right)$ as a smoothing function. Introducing a smoothed density of the single-particle alignment by

$$
\tilde{\rho}_{\mathrm{al}}^{(\nu, \pi)}(\lambda)=\sum_{n \in \nu, \pi} i_{n}^{2} g\left(\lambda-e_{n}\right)
$$

as a function of the Fermi energy $\lambda$, the dispersion $\Delta \omega_{\text {therm }}$ of rotational frequency can be expressed in terms of the smoothed single-particle alignment,

$$
\begin{aligned}
\Delta \omega_{\text {therm }} & =\sqrt{\left(\Delta \omega^{(\nu)}\right)^{2}+\left(\Delta \omega^{(\pi)}\right)^{2}}, \\
\Delta \omega^{(\nu, \pi)} & =\frac{1}{\mathcal{J}} \sqrt{T \tilde{\rho}_{\mathrm{al}}^{(\nu, \pi)}\left(\lambda_{\nu, \pi}\right)},
\end{aligned}
$$

where $\Delta \omega^{(\nu)}$ and $\Delta \omega^{(\pi)}$ represent the neutron and proton contributions to $\Delta \omega_{\text {therm }}$, respectively. They are plotted in Fig. 8 as functions of the Fermi energy $\lambda$. 


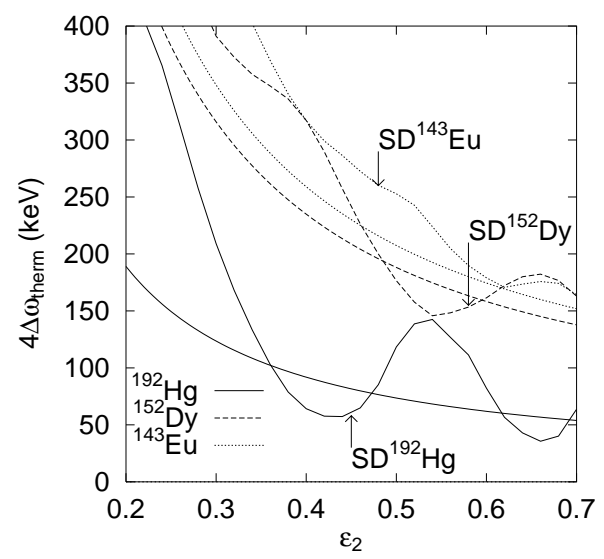

Figure 9: The dispersion of rotational frequency $4 \Delta \omega_{\text {therm }}$ as a function of quadrupole deformation parameter $\epsilon_{2}$ at $I=40 \hbar$ for ${ }^{192} \mathrm{Hg}$ and $I=50 \hbar$ for the others. Temperature is the same as Fig.7. The analytic estimate $4 \Delta \omega_{\text {h.o. }}$ showing the $\epsilon_{2}^{-1}$ dependence is also plotted for the sake of comparison. The equilibrium deformation of superdeformed states is indicated by arrows.

The quantity $\Delta \omega^{(\nu, \pi)}$ (and the alignment density $\tilde{\rho}_{a}^{(\nu, \pi)}$ ) shows oscillation as a function of the Fermi energy with a typical period of $5 \mathrm{MeV}$. Maximum points of the oscillation are related to aligned high- $j$ or high- $N$ orbits. Taking $\Delta \omega^{(\nu)}$ as an example, the maximum at $\lambda \sim 46 \mathrm{MeV}$ is caused by the lowest and most aligned $N=7$ orbit $\left(7_{1}\right)$ and the second $7_{2}$ orbit (which originate from $j_{15 / 2}$ ), and the next peak at $\lambda \sim 51 \mathrm{MeV}$ corresponds to $8_{1}$ (and $8_{2}$ ). On the other hand, $\Delta \omega^{(\nu)}$ takes locally minimum value at around $\lambda \sim 49 \mathrm{MeV}$, where there exist no highly aligned orbits, but instead present are the oblate orbits with high- $\Omega$ value such as $[514] \frac{7}{2},[512] \frac{5}{2},[505] \frac{11}{2}$ which have little alignments $\left(i_{n} \approx 0\right)$. It should be noticed that the neutron Fermi surface of superdeformed ${ }^{192} \operatorname{Hg}(N=112)$ is located near the minimum point of the shell oscillation. The proton contribution $\Delta \omega^{(\pi)}$ also shows a similar shell oscillation, and the proton Fermi surface of superdeformed ${ }^{192} \mathrm{Hg}(Z=80)$ is also situated near the minimum position of the shell oscillation. In Fig/8, the smoothed spectral density of single-particle routhians $e_{n}$, i.e., $\tilde{\rho}^{(\nu, \pi)}(\lambda)=\sum_{n \in \nu, \pi} g\left(\lambda-e_{n}\right)$, is also plotted. From the comparison with $\tilde{\rho}_{\mathrm{al}}^{(\nu, \pi)}$, it is seen that the shell oscillation of the alignment density is not directly related to the spectral density, but mainly reflects the single-particle alignments $i_{n}$.

One can easily expect that the maxima and minima of the alignment density (dispersion of rotational frequency) varies strongly as a function not only of the Fermi energy but also of deformation and rotational frequency since the routhian energy of the strongly aligned orbits generally depends on these quantities. The non-linear spin dependence of $\Delta \omega$ seen in Fig.7 is originated from the shell effect in the single-particle alignments. Similar non-linear spin dependence due to aligned orbits was also recognized in the calculation of $\Delta \omega_{\text {therm }}$ in normal deformed ${ }^{168} \mathrm{Yb}$ [10, 22]. The dispersion $\Delta \omega_{\text {therm }}$ of rotational frequency calculated as a function of quadrupole deformation parameter(Fig.9) also shows significant oscillation. In Fig.9, we plot for comparison an analytic estimate [2, 10] of $\Delta \omega$ based on the harmonic potential model

$$
4 \Delta \omega_{\text {h.o. }}=290.2 A^{-29 / 12} \epsilon^{-1} I E_{\mathrm{ex}}^{1 / 4}[\mathrm{MeV}],
$$

which neglects the shell effects and therefore gives an average (and smooth) behavior. The comparison indicates that the shell effect of alignment is the same order of magnitude as the average value. In ${ }^{192} \mathrm{Hg}$ the shell effect suppresses the dispersion of rotational frequency at the deformation (and Fermi surface) of the superdeformed states. Note that different situation is realized in superdeformed nuclei in $A \sim 150$ region, where the Fermi surface is located in the vicinity of the aligned high- $N$ orbits such as $\nu 7_{1}$ and $\nu 7_{2}$. This enhances $\Delta \omega$ in ${ }^{143} \mathrm{Eu}$ as shown in Fig.9.

Thus the shell structure in the single-particle alignment influences significantly the dispersion of rotational frequency $\Delta \omega$, and consequently this causes very small rotational damping width $\Gamma_{\text {rot }}$ in 
superdeformed ${ }^{192} \mathrm{Hg}$.

\subsection{Large number of bands and retarded onset of damping}

In this subsection, we discuss the mechanism for the very large number of rotational superdeformed bands $\left(N_{\text {band }} \sim 150\right)$ obtained in ${ }^{192} \mathrm{Hg}$.

In the preceding paper [6], we showed that the relatively large number of bands $N_{\text {band }} \sim 40-$ 100 seen in the calculation for $A \sim 150$ superdeformed nuclei(compared with the value $N_{\text {band }} \sim$ 30 for rare-earth normally deformed nuclei) is due to the level density effect associated with the superdeformed shell gap in the single-particle routhian spectrum. This mechanism, however, does not account for the calculated results for ${ }^{192} \mathrm{Hg}$.

To show this, let us repeat briefly the arguments for the level density effect [6]. In that argument we assumed that the onset of rotational damping takes place as soon as the configuration mixing among basis $n \mathrm{p}-n \mathrm{~h}$ states becomes strong. The condition for configuration mixing may be expressed as $\Gamma_{\mu}>1 / \rho_{2 \text { body }}$ in terms of the spreading width $\Gamma_{\mu}$ of the basis $n \mathrm{p}-n \mathrm{~h}$ states and the density $\rho_{2 \text { body }}$ of $n \mathrm{p}-n \mathrm{~h}$ states which can interact via the two-body interaction [2]. Assuming the Fermi golden rule for the spreading width $\Gamma_{\mu}=2 \pi \rho_{2 \text { body }} \bar{v}^{2}$, the condition can be written also in terms of the average matrix element $\bar{v}$ of the residual two-body interaction and the spacing $d_{2}=1 / \rho_{2 \text { body }}$ between interactive states,

$$
d_{2}<\sqrt{2 \pi} \bar{v}
$$

If we assume the level density $\rho$ and two-body level density $\rho_{\text {2body }}$ of $n \mathrm{p}-n \mathrm{~h}$ states follow the Fermi gas formula [23, 24,

$$
\begin{gathered}
\rho(E)=\frac{\sqrt{\pi}}{48} a^{-\frac{1}{4}} E^{-\frac{5}{4}} \exp 2 \sqrt{a E}, \\
\rho_{2 \text { body }}(E)=\frac{81}{4 \pi^{6}} a^{5 / 2} E^{3 / 2},
\end{gathered}
$$

the onset energy of configuration mixing (and rotational damping) is expressed in terms of the level density parameter $a$ and the average matrix element $\bar{v}$ as $E_{\text {onset }} \propto \bar{v}^{-2 / 3} a^{-5 / 3}$ [6]. Similarly the number of rotational bands is expressed as $N_{\text {band }}=\int{ }^{E_{\text {onset }}} \rho d E$, which is a monotonically increasing function of $a E_{\text {onset }}$ and hence of $(a \bar{v})^{-1}$.
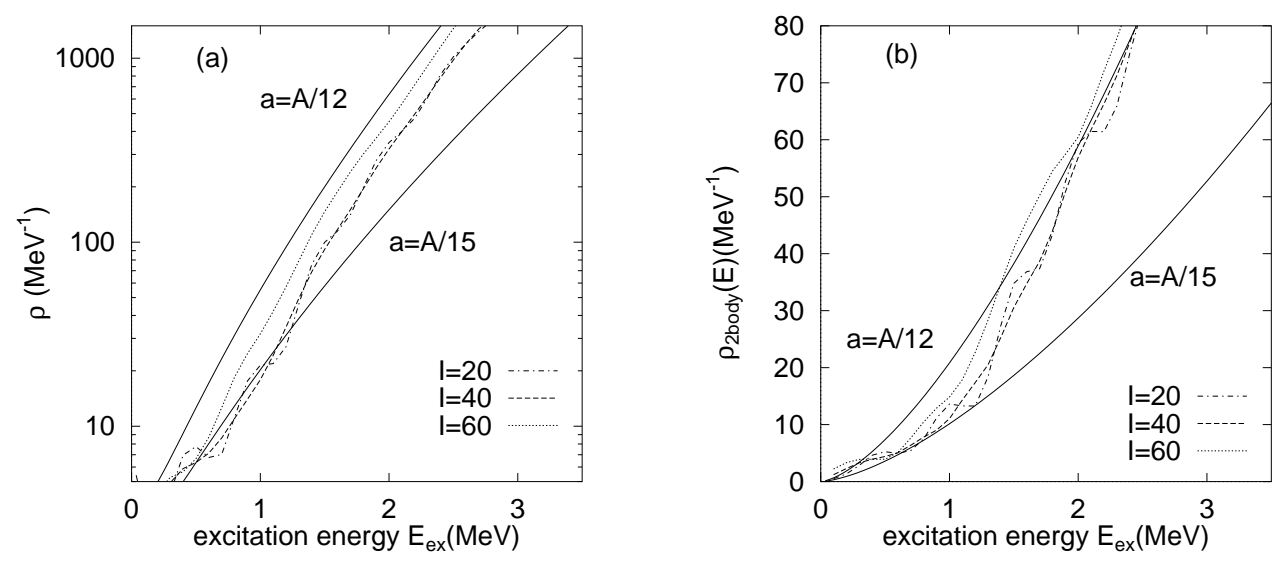

Figure 10: (a)The total level density and (b)the two-body level density of unperturbed manyparticle many-hole states for superdeformed ${ }^{192} \mathrm{Hg}$ at spins $I=20,40,60 \hbar$. Here average is taken over both parities and signatures, i.e., $I^{ \pm}, I+1^{ \pm}$. The Fermi gas formulas with the level density parameters $a=A / 12$ and $A / 15$ are also plotted.

The level density parameter $a$ is usually parameterized as $a=A / a_{0}$ where $a_{0} \sim 10 \mathrm{MeV}$ for normally deformed nuclei in the cranked Nilsson model. For the superdeformed states, the level 


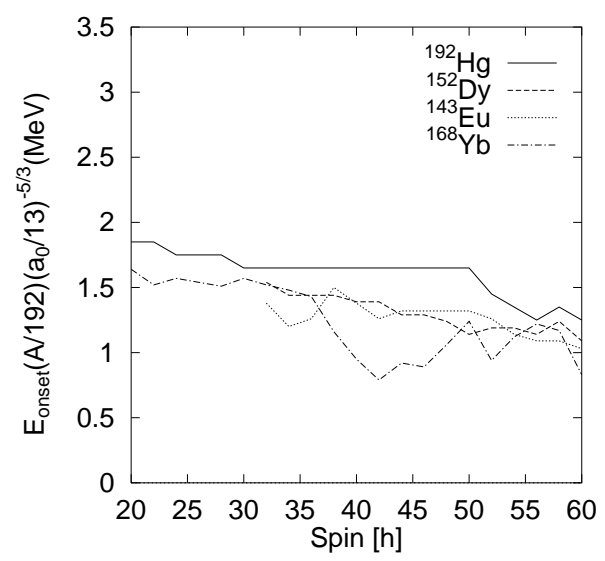

Figure 11: The onset energy of rotational damping $E_{\text {onset }}$ for superdeformed ${ }^{192} \mathrm{Hg},{ }^{152} \mathrm{Dy},{ }^{143} \mathrm{Eu}$ and normally deformed ${ }^{168} \mathrm{Yb}$ multiplied by a factor $(A / 192)\left(a_{0} / 13\right)^{-5 / 3}$, which removes the level density effect. We adopt $a_{0}=13$ for ${ }^{192} \mathrm{Hg}$ and $a_{0}=17,15,10$ for ${ }^{152} \mathrm{Dy},{ }^{143} \mathrm{Eu}$ and ${ }^{168} \mathrm{Yb}$, respectively.

density is smaller than that of normally deformed states because of the shell gap in the singleparticle routhian spectrum, and this can be represented by larger value of $a_{0}$, which is estimated as $a_{0}=15,17 \mathrm{MeV}$ for ${ }^{143} \mathrm{Eu}$ and ${ }^{152} \mathrm{Dy}$, respectively [6]. Calculated level densities of superdeformed states for ${ }^{192} \mathrm{Hg}$ is shown in Fig.10, from which $a_{0} \sim 13 \mathrm{MeV}$ is estimated. On the other hand, the average magnitude $\bar{v}$ of the two-body elements $v_{i j k l}=\int d \vec{x} d \vec{x}^{\prime} v_{\tau} \delta\left(\vec{x}-\vec{x}^{\prime}\right) \phi_{i}^{*} \phi_{j}^{*} \phi_{k} \phi_{l}$ is estimated as $\bar{v} \sim$ (volume integral of the two-body force)/(nuclear volume) $\propto A^{-1}$. The r.m.s value of the calculated off-diagonal matrix elements $v_{i j k l}$ is $\bar{v}=20,18,12$ and $16 \mathrm{keV}$ for ${ }^{143} \mathrm{Eu},{ }^{152} \mathrm{Dy},{ }^{192} \mathrm{Hg}$, and normally deformed ${ }^{168} \mathrm{Yb}$. They approximately follow the expected $A^{-1}$ dependence. Using the above characterizations of the level density and the residual interaction, the dependence of $E_{\text {onset }}$ and $N_{\text {band }}$ on the level density shell effect (parameter $a_{0}$ ) and the mass number $A$ is estimated as $E_{\text {onset }} \propto A^{-1} a_{0}^{-5 / 3}$, and $N_{\text {band }}$ is an monotonically increasing function of $a_{0}$ and independent of $A$. This argument predicts that $N_{\text {band }}$ in ${ }^{192} \mathrm{Hg}$ would be smaller than those in ${ }^{152} \mathrm{Dy}$ and ${ }^{143} \mathrm{Eu}$.

It is noted, however, that microscopically calculated $N_{\text {band }}$ for superdeformed ${ }^{192} \mathrm{Hg}$ is much larger than those for ${ }^{152} \mathrm{Dy}$ and ${ }^{143} \mathrm{Eu}$ (Fig.2(a)). This indicates that the excessively large number of superdeformed rotational bands in ${ }^{192} \mathrm{Hg}$ cannot be explained just by the shell effect in the level density. This is also demonstrated in Fig 11 which plots the microscopically calculated $E_{\text {onset }}$ multiplied by a factor $(A / 192)\left(a_{0} / 13\right)^{-5 / 3}$ with which we intend to remove the level density shell effect. It is seen that the scaled onset energy in ${ }^{192} \mathrm{Hg}$ is higher than that for ${ }^{143} \mathrm{Eu},{ }^{152} \mathrm{Dy}$ and ${ }^{168} \mathrm{Yb}$. If we assume the above argument the onset energy of configuration mixing is estimated as $E_{x}=1.2 \sim 1.3 \mathrm{MeV}$ for ${ }^{192} \mathrm{Hg}$ with use of the scaling property $\propto A^{-1} a_{0}^{5 / 3}$ and $E_{\text {onset }}=800 \mathrm{keV}$ and $a_{0}=10 \mathrm{MeV}$ [5] for normally deformed ${ }^{168} \mathrm{Yb}$ whereas the microscopically calculated onset energy of rotational damping is about $1.6 \mathrm{MeV}$.

The above analysis indicates that the onset of rotational damping in ${ }^{192} \mathrm{Hg}$ takes place at higher excitation energy $\left(E_{x} \sim 1.6 \mathrm{MeV}\right)$ than onset energy $\left(E_{x} \sim 1.2 \mathrm{MeV}\right)$ of configuration mixing of $n \mathrm{p}-n$ h states. Namely, the onset of rotational damping is slightly "retarded" in comparison with the onset of mixing. This indicates that the mechanism of the onset of rotational damping is qualitatively different from those in the normal deformed rare-earth nuclei and $A \sim 150$ superdeformed nuclei.

To understand the mechanism of onset of rotational damping in ${ }^{192} \mathrm{Hg}$, it is useful to recall the argument 25, 26] that discusses possible occurrence of 'ergodic rotational bands' which means rotational band structures formed by compound intrinsic states (the states whose wave functions have strong configuration mixing). The ergodic rotational bands are expected to realize when the rotational damping width is smaller than the level spacing $d=1 / \rho$ of the all levels at fixed $I^{\pi}$,

$$
\Gamma_{\text {rot }}<d
$$


while wave functions of the energy levels are compound mixture of basis many-particle many-hole states. In this situation, a compound state at spin $I$ decays by E2 transition to a single final compound state at $I-2$, and hence sequence of such $\mathrm{E} 2$ transitions forms a band structure in spite of the strong mixing.

In the calculated situation for ${ }^{192} \mathrm{Hg}$, many of the excessive number of rotational bands reside in the energy region between $E_{x} \sim 1.2 \mathrm{MeV}$ (the estimated onset energy of mixing, see above) and $E_{x} \sim 1.6 \mathrm{MeV}$ (the onset energy of rotational damping). Many of the energy levels in this energy region are some mixture of basis many-particle many-hole configurations although they are not fully ergodic since their excitation energy is just a few hundred keV above the onset energy of configuration mixing [27]. The rotational damping width $\Gamma_{\text {rot }}$ in ${ }^{192} \mathrm{Hg}$ is evaluated to be $20-30 \mathrm{keV}$ for spins $I<50$ (Fig.6). The total level spacing $d$ is $\sim 30 \mathrm{keV}$ at $E_{x}=1.2 \mathrm{MeV}$, and $\sim 10 \mathrm{keV}$ at $E_{x}=1.6 \mathrm{MeV}$ (estimated from Fig.10(a)). Namely, the rotational damping width is comparable with the level spacing,

$$
\Gamma_{\text {rot }} \sim d
$$

in the energy region under discussion. Thus, the situation lies around the borderline of the condition Eq.(21) for the ergodic rotational bands. This suggests that similar mechanism to that of the ergodic rotational bands is taking place in superdeformed ${ }^{192} \mathrm{Hg}$, and explains why the energy levels in the region of $E_{x} \sim 1.2-1.6 \mathrm{MeV}$ form rotational band structures and contribute to the large number of $N_{\text {band }}$. The above argument explains also the observation that $N_{\text {band }}$ in ${ }^{192} \mathrm{Hg}$ decreases as spin increases, especially for $I>50$, since $\Gamma_{\text {rot }}$ becomes as large as $\Gamma_{\text {rot }} \gtrsim 30 \mathrm{keV}$ for $I \geq 50 \hbar$, which tends to violate the relation Eq.(22). Note that the above mechanism does not manifest itself in normally deformed ${ }^{168} \mathrm{Yb}$ and superdeformed ${ }^{143} \mathrm{Eu}$ where $\Gamma_{\text {rot }}$ is much larger. For superdeformed ${ }^{152} \mathrm{Dy}$, the mechanism may be relevant to explain the small spin dependence of $N_{\text {band }}$ seen in Fig.2(b) although the effect is not very significant (cf Ref.[6]). It should be recalled here that the shell effect associated with the single-particle alignment plays an important role in causing such small $\Gamma_{\text {rot }}$ particularly in superdeformed ${ }^{192} \mathrm{Hg}$, but not in other calculated nuclei, as we discussed in the previous subsection.

\section{Conclusions}

We performed microscopic description of the rotational damping associated with superdeformed states in ${ }^{192} \mathrm{Hg}$. We obtained remarkably large number of superdeformed rotational bands, $N_{\text {band }} \sim$ 150 and very small rotational damping width $\Gamma_{\text {rot }} \sim 30 \mathrm{keV}$, which are different by more than factor 2 from those in $A \sim 150$ superdeformed nuclei and rare-earth normally deformed nuclei. It is also found that the rotational damping width varies in different nuclei in the $A \sim 150$ region. The strong variation of the rotational damping width is attributed to the shell structure associated with the angular momentum alignment of the single-particle orbits in the cranked Nilsson potential. The alignments of the excited many-particle many-hole configurations have different values for different states. Their dispersion, which is strongly related to the rotational damping width, depends on the presence of highly aligned orbits in the vicinity of the Fermi surface.

We pointed out that in superdeformed ${ }^{192} \mathrm{Hg}$ the very small rotational damping width due to the shell effect causes slightly different mechanism for the onset of rotational damping. In the cases of the rare-earth normally deformed nuclei and $A \sim 150$ superdeformed nuclei, the onset of rotational damping takes place at the same energy as the onset energy of configuration mixing among $n \mathrm{p}-n \mathrm{~h}$ states. In ${ }^{192} \mathrm{Hg}$, on the other hand, energy levels in the onset region of configuration mixing do not necessarily accompany damped rotational decay since the damping width is as small as the level spacing of the final states. This leads to the significantly large number of superdeformed rotational bands in ${ }^{192} \mathrm{Hg}$.

Summarizing the present investigations together with those in the preceding paper [6], we revealed two kinds of shell effects which affect properties of the rotational damping associated with superdeformed states. Firstly, because of the shell gap in the single-particle spectrum of the rotating superdeformed mean field, the level density of excited superdeformed states is relatively low and the configuration mixing is weak compared with normally deformed nuclei. This influences onset properties of the rotational damping as demonstrated by the calculations for $A \sim 150$ region [6]. The second one, which was discussed in the present paper, is originated from the shell structure in the 
angular momentum alignments of the single-particle orbits. It affects strongly the damping width of rotational damping, but also modifies the onset properties of the damping under certain conditions as seen in ${ }^{192} \mathrm{Hg}$.

\section{Acknowledgment}

The authors would like to thank K. Matsuyanagi, T. Døssing, and E. Vigezzi for helpful discussions.

\section{References}

[1] G.A. Leander, Phys. Rev.C25(1982)2780.

[2] B. Lauritzen, T. Døssing, and R.A. Broglia, Nucl. Phys. A457(1986)61.

[3] B. Herskind, A. Bracco, R.A. Broglia, T. Døssing, A. Ikeda, S. Leoni, J. Lisle, M. Matsuo, and E. Vigezzi, Phys. Rev. Lett. 68(1992)3008;

T. Døssing, B. Herskind, S. Leoni, M. Matsuo, A. Bracco, R.A. Broglia, and E. Vigezzi, Physics Reports 268(1996) 1.

[4] S.Åberg, Phys. Rev. Lett. 64(1990)3119;

S. Åberg, Prog. Part. Nucl. Phys. vol.28 (Pergamon, 1992)p11.

[5] M. Matsuo, T. Døssing, E. Vigezzi, R.A. Broglia, and K. Yoshida, Nucl. Phys. A617(1997)1.

[6] K. Yoshida and M. Matuso, Nucl. Phys. A612(1997)26.

[7] K. Schiffer and B. Herskind, Phys. Lett. B255 (1991)508;

K. Schiffer and B. Herskind, Nucl. Phys. A520(1990)521c.

[8] S. Leoni, B. Herskind, T. Døssing, K. Yoshida, M. Matsuo, A. Ataç, G.B. Hagemann, F. Ingebresten, H.J. Jensen, R.M. Lieder, G.V. Marti, N. Nica, J. Nyberg, M. Piparinen, H. Schnare, G. Sletten, K. Stähle, M. Sugawara, P.O. Tjøm, and A. Virtanen, Phys. Lett. B353(1995)179.

[9] T. Lauritsen, Ph. Benet, T.L. Khoo, K.B. Beard, I. Ahmad, M.P. Carpenter, P.J. Daly, M.W. Drigert, U. Grag, P.B. Fernandez, R.V.F. Janssens, E.F.Moore, F.L.H. Wolfs, and D. Ye, Phys.Rev.Lett. 69(1992)2479.

[10] T. Døssing and B. Herskind, Proc. Intern. Symp. on New Trends in Nuclear Collective Dynamics (Nishinomiya) (Springer-Verlag, 1992)p239

[11] T. Bengtsson, Nucl. Phys. A496(1989)56.

[12] G. Anderson, S.E. Larsson, G. Leander, P. Møller , S.G. Nilsson, I. Ragnarsson, S. Åberg, R. Bengtsson, J. Dudek, B. Nerlo-Pomorska, K. Pomorski, and Z. Szymanski, Nucl. Phys. A268(1976)205.

[13] M. Brack, J. Damgaad, A.S. Jensen, H.C. Pauli, V.M. Strutinsky, C.Y. Wong, Rev.Mod.Phys. 44(1972)320.

[14] W.D. Myers and W.J. Swiatecki, Arkiv Fysik 36(1967)343 ;

S. Cohen, F. Plasil and W.J. Swiatecki, Ann. Phys. 82(1974)557.

[15] B.W. Bush, G.F. Bertsch, and B.A. Brown, Phys. Rev. C45(1992)1709.

[16] E. Vigezzi, R.A. Broglia and T. Døssing, Phys. Lett. 249(1990), 163;

E. Vigezzi, R.A. Broglia and T. Døssing, Nucl. Phys. A520(1990), 179c. 
[17] Y.R. Shimizu, F. Barranco, R.A. Broglia, T. Døssing and E. Vigezzi, Phys. Lett. B274(1992), 243.

[18] B. Herskind, T. Døssing, D. Jerrestam, K. Schiffer, S. Leoni, J. Lislie, R. Chapman, F. Khazaie and J.N. Mo, Phys. Lett. B276 (1992) 4;

B. Herskind, T. Døssing, S. Leoni, M. Matsuo, N. Nica, D.C. Radford and P. Rasmussen, Nucl. Phys. A557 (1993) 191c.

[19] B. Herskind, T. Døssing, S. Leoni, M. Matsuo, and E. Vigezzi, Prog. Part. Nucl. Phys. Vol.28 (Pergamon 1992) p.235

[20] S. Leoni, B. Herskind, T. Døssing, P. Rasmussen, P. Bosetti, A. Bracco, R. Broglia, S. Frattini, M. Matsuo, N. Nica, E. Vigezzi, A. Ataç, M. Bergström, A. Brockstedt, H. Carlssont, P. Ekströmt, F. Ingebretsen, H.J. Jensen, J. Jongman, G.B. Hagemann, R.M. Lieder, T. Lönnroth, A. Maj, B. Million, A. Nordlund, J. Nyberg, M. Piiparinen, H. Ryde, M. Sugawara, P.O. Tjøm, and A. Virtanen, Nucl. Phys. A587 (1995) 513.

[21] R.A. Broglia, T. Døssing, M. Matsuo, E. Vigezzi, P. Bosetti, A. Bracco, S. Frattini, B. Herskind, S. Leoni, and P. Rasmussen, Z. Phys. A356(1996)259.

[22] B. Herskind, T.Døssing, S. Leoni, M.Matsuo, N. Nica, D.C. Radford and P.Rasmussen, Nucl. Phys. A557(1993)191c.

[23] A. Bohr and B.R. Mottelson, Nuclear Structure. vol.1 (Benjamin, 1969).

[24] S. Åberg, Nucl. Phys. A477(1988)18.

[25] B.R. Mottelson, Nucl. Phys. A557(1993)717c;

B.R.Mottelson, Proc. The Frontier of Nuclear Spectroscopy(Kyoto)(World Scientific, 1992)p7.

[26] S. Aberg, Z. Phys. A358(1997)269;

S.Aberg, Lund-MPh-96/02, preprint

[27] M. Matsuo, T. Døssing, E. Vigezzi and S. Aberg, Nucl. Phys. A620(1997)296. 